\title{
Role of adjuvant or salvage radiosurgery in the management of unresected residual or progressive glioblastoma multiforme in the pre-bevacizumab era
}

\author{
Ajay Niranjan, MCh, MBA, ${ }^{1,3}$ Hideyuki Kano, MD, PhD, ${ }^{1,3}$ Aditya lyer, MD, MEng, ${ }^{4}$ \\ Douglas Kondziolka, MD, ${ }^{5}$ John C. Flickinger, MD, ${ }^{1-3}$ and L. Dade Lunsford, MD ${ }^{1-3}$
}

Departments of ${ }^{1}$ Neurological Surgery and ${ }^{2}$ Radiation Oncology, and ${ }^{3}$ Center for Image-Guided Neurosurgery, ${ }^{4}$ University of Pittsburgh School of Medicine, Pittsburgh, Pennsylvania; and ${ }^{5}$ Department of Neurological Surgery, New York University, New York, New York

OBJECT After initial standard of care management of glioblastoma multiforme (GBM), relatively few proven options remain for patients with unresected progressive tumor. Numerous reports describe the value of radiosurgery, yet this modality appears to remain underutilized. The authors analyzed the outcomes of early adjuvant stereotactic radiosurgery (SRS) for unresected tumor or later salvage SRS for progressive GBM. Radiosurgery was performed as part of the multimodality management and was combined with other therapies. Patients continued to receive additional chemotherapy after SRS and prior to progression being documented. In this retrospective analysis, the authors evaluated factors that affected patient overall survival (OS) and progression-free survival.

METHODS Between 1987 and 2008 the authors performed Gamma Knife SRS in 297 patients with histologically proven GBMs. All patients had received prior fractionated radiation therapy, and $66 \%$ had undergone one or more chemotherapy regimens. Ninety-six patients with deep-seated unresectable GBMs underwent biopsy only. Of those in whom excision had been possible, resection was considered to be gross total in 68 and subtotal in 133. The median patient age was 58 years (range 23-89 years) and the median tumor volume was $14 \mathrm{~cm}^{3}$ (range $0.26-84.2 \mathrm{~cm}^{3}$ ). The median prescription dose delivered to the imaging-defined tumor margin was $15 \mathrm{~Gy}$ (range 9-25 Gy). The median follow-up duration was 8.6 months (range 1.1-173 months). Cox regression models were used to analyze survival outcomes. Variables examined included age, residual versus recurrent tumor, prior chemotherapy, time to first recurrence, SRS dose, and gross tumor volume.

RESULTS The median survival times after radiosurgery and after diagnosis were 9.03 and 18.1 months, respectively. The 1-year and 2-year OS after SRS were 37.9\% and 16.7\%, respectively. The 1-year and 2-year OS after diagnosis were $76.2 \%$ and $30.8 \%$, respectively. Using multivariate analysis, factors associated with improved OS after diagnosis were younger age (<60 years) at diagnosis $(p<0.0001)$, tumor volume $<14 \mathrm{~cm}^{3}(p<0.001)$, use of prior chemotherapy $(p=0.001)$, and radiosurgery at the time of recurrence $(p<0.0001)$. Multivariate analysis showed that younger age $(p$ $<0.0001)$ and smaller tumor volume $\left(<14 \mathrm{~cm}^{3}\right)(p=0.001)$ were significantly associated with increased OS after SRS. Adverse radiation effects were seen in 69 patients (23\%). Fifty-eight patients (19.5\%) underwent additional resection after SRS. The median survivals after diagnosis for recursive partitioning analysis Classes III, IV and V+VI were 31.6, 20.8 , and 16.7 months, respectively.

CONCLUSIONS In this analysis 30\% of a heterogeneous cohort of GBM patients eligible for SRS had an OS of 2 years. Radiosurgery at the time of tumor progression was associated with a median survival of 21.8 months. The role of radiosurgery for GBMs remains controversial. The findings in this study support the need for a funded and appropriately designed clinical trial that will provide a higher level of evidence regarding the future role of SRS for glioblastoma patients in whom disease has progressed despite standard management.

http://thejns.org/doi/abs/10.3171/2014.11.JNS13295

KEY WORDS glioblastoma multiforme; stereotactic radiosurgery; Gamma Knife; oncology

ABBREVIATIONS ARE = adverse radiation effect; GBM = glioblastoma multiforme; KPS = Karnofsky Performance Scale; MST = median survival time; OS = overall survival; PFS = progression-free survival; RPA = recursive partitioning analysis; RT = radiation therapy; RTOG = Radiation Therapy Oncology Group; SRS = stereotactic radiosurgery.

SUBMITTED December 2, 2013. ACCEPTED November 18, 2014

INCLUDE WHEN CITING Published online January 16, 2015; DOI: 10.3171/2014.11.JNS13295.

DISCLOSURE Dr. Lunsford reports being a consultant for and owning stock in Elekta AB. 
$\mathrm{G}$ LIOBLASTOMA multiforme (GBM) is the most common primary brain tumor in adults, with nearly 10,000 cases diagnosed annually in the United States. Unfortunately, despite surgery, adjuvant fractionated radiation therapy (RT), and chemotherapy, the prognosis remains poor. Survival is improved when patients have tumors located in brain areas that allow for maximal resection..$^{3,8,10,43}$ Although multimodality management remains largely palliative, median survival times (MSTs) of 14.6 months are now possible. Conventional fractionated RT, to cumulative doses of $60 \mathrm{~Gy}$, is required to improve survival rates, ${ }^{1,46}$ but further dose escalation fails to improve outcomes. Because of the infiltrative nature of glioblastoma, regional recurrence within $2 \mathrm{~cm}$ of the initial tumor remains a potent cause of relapse. ${ }^{20,47}$

Across the world Gamma Knife (Elekta AB) stereotactic radiosurgery (SRS) has been used as an adjuvant therapy in more than 40,000 patients with glial neoplasms. Although prior retrospective studies have documented a modest survival advantage with the addition of SRS delivered using various technologies, ${ }^{14,23,24,27,29,32,34,39}$ others have found no added benefit. ${ }^{4,9,16,37,38}$ A single prospective randomized trial by the Radiation Therapy Oncology Group (RTOG 93-05) ${ }^{42}$ found no improvement in overall survival (OS) when various forms of linear acceleratorand Gamma Knife-based SRS were given prior to conventional fractionated RT. ${ }^{15}$ That study was not designed to evaluate the potential role of SRS at the time of GBM progression in patients who had received standard of care initial management. The present retrospective report evaluates our single-center SRS experience with glioblastoma patients who underwent multimodality management before the availability of bevacizumab. SRS was added as an option to the treatment plan for patients with unresected tumors and for patients at the time of tumor progression. A small series of patients in whom adjuvant bevacizumab was used was recently published and those patients are not included in the present report. ${ }^{30}$

\section{Methods}

\section{Patient Population}

We performed a retrospective outcome analysis of 297 consecutive patients with histologically proven GBM. All patients underwent SRS that involved one or more Leksell Gamma Knife SRS technologies between 1987 and 2008. This population represented patients treated in the prebevacizumab era. In 96 patients tumors were deep seated and in 201 patients they were lobar. Patients with tumors in accessible lobar locations underwent initial gross-total resection $(n=68)$ or subtotal $(<90 \%$ tumor $)$ resection $(n=$ 133). The 96 patients ineligible for cytoreductive surgery (that is, those whose tumors were located in deep locations such as the basal ganglia, thalamus, or brainstem) underwent stereotactic biopsy. All patients then received fractionated external-beam RT (median dose 60.0 Gy in daily 2.0-Gy fractions). Overall, 197 patients (66\%) received concomitant chemotherapy. Patients were referred for adjuvant SRS early in their treatment course if they had imaging evidence of surgically unresected residual disease $(n=144)$ or later in the treatment course when tumor progression was documented despite standard-ofcare management $(n=153)$.

Tumor progression was identified using serial imaging (both T1-weighted contrast-enhanced and T2-weighted MRI). Patients were eligible for SRS if there was early evidence of unresected tumor or delayed evidence of a progressing tumor. All patients had Karnofsky Performance Scale (KPS) scores $\geq 60$. A total of 323 SRS procedures were performed. The median patient age was 58 years (23-89 years).

All patients had serial clinical and imaging evaluations at 10 weeks after SRS and then at 3-month intervals thereafter. Symptomatic adverse radiation effects (AREs) were defined as the development of new neurological symptoms or signs in the absence of overt tumor progression. The University of Pittsburgh institutional review board approved this single-institution, retrospective study. The primary end points of the study were OS after diagnosis and progression-free survival (PFS) after SRS.

\section{Radiosurgical Technique}

Our radiosurgical technique has been described in detail in previous reports. ${ }^{28}$ In brief, patients underwent application of an imaging-compatible stereotactic head frame followed by CT scanning or high-resolution MRI. The radiosurgery target prescription volume included the contrast-enhanced tumor regions. Gross tumor volume was defined as the paramagnetic contrast-enhanced tumor edge on T1-weighted MR images. It was included in the planned isodose volume. The median gross tumor volume was $14 \mathrm{~cm}^{3}\left(0.26-84.2 \mathrm{~cm}^{3}\right)$. The median prescription dose delivered to the tumor margin was 15.0 Gy (9-25 Gy). The median maximum dose was 26.0 Gy (range 20$50 \mathrm{~Gy})$. The median follow-up duration was 8.6 months (range 1.1-173 months). SRS was delivered in a single procedure using Leksell Gamma Knife models U, B, C, 4C, or Perfexion.

\section{Statistical Analysis}

The outcome measures considered were OS after initial diagnosis, OS after SRS, and PFS after SRS. For survival after diagnosis, we used the date of diagnosis until death or last follow-up. The OS after SRS was calculated from the day of the SRS until day of death or last follow-up using the Kaplan-Meier method. We constructed KaplanMeier plots for PFS using the dates of SRS and follow-up MRIs. Univariate analysis was performed using log-rank statistics with $\mathrm{p}<0.05$ set as significant. Variables that were considered to be clinically relevant to prognosis were included in the multivariate models. These variables included patient age, tumor volume, margin dose, extent of initial resection, deep versus lobar location, time between initial diagnosis and SRS, radiosurgery for residual versus recurrent tumor, use of chemotherapy, and KPS. Cox regression models were used to analyze these outcomes. The results of multivariate Cox models are reported as ORs, 95\% CIs, and p values. Standard statistical processing software (SPSS, version 15.0, and Prism, version 4.0) was used. 


\section{Results}

\section{Overall Survival After Diagnosis}

At the time of analysis, 265 patients (89\%) had died. The MST from the date of diagnosis (on the basis of KaplanMeier estimates) was 18.1 months (95\% CI 16.86-19.34 months). Multivariate analysis showed that younger age (< 60 years) at diagnosis $(\mathrm{p}=0.0001)$, tumor volume $<14 \mathrm{~cm}^{3}$ $(p=0.001)$, use of any chemotherapy $(p=0.001)$, and SRS at the time of recurrence $(\mathrm{p}<0.0001)$ were significantly associated with improved OS after diagnosis.

Median OS after diagnosis was significantly better ( $p$ $<0.0001$ ) for patients who underwent SRS for progressive tumors than for those who underwent early SRS for unresected tumor volumes. The MST for patients who underwent later SRS at the time of progression was 21.8 months, whereas it was 14.1 months for patients who underwent early SRS for unresected tumors (Fig. 1).

Univariate analysis showed that the median OS after diagnosis was significantly better $(p=0.007)$ for patients who had lobar tumors eligible for resection than for patients who had unresectable deep-seated tumors. The MST for patients with resected lobar tumors was 19.1 months (95\% CI 17.51-20.69 months) compared with 16.1 months for patients with unresectable deep-seated tumors (95\% CI 13.83-18.36 months).

\section{Overall Survival After SRS}

The overall MST after SRS was 9.03 months (95\% CI 8.1-10.0 months). In the univariate analysis, smaller tumor volume, higher margin dose, SRS for recurrent tumors, post-SRS chemotherapy (vs no chemotherapy), post-SRS temozolomide (vs other chemotherapy agent or no chemotherapy), and resection after SRS were significantly associated with longer survival after SRS.

The MST for patients with tumor volumes $<14 \mathrm{~cm}^{3}$ was 11.2 months (95\% CI 9.4-13.1 months). For patients with tumor volumes $<14 \mathrm{~cm}^{3}$, the 6-month, 1-year, 2-year, 3 -year, and 5-year survival rates after SRS were $80.4 \%$,

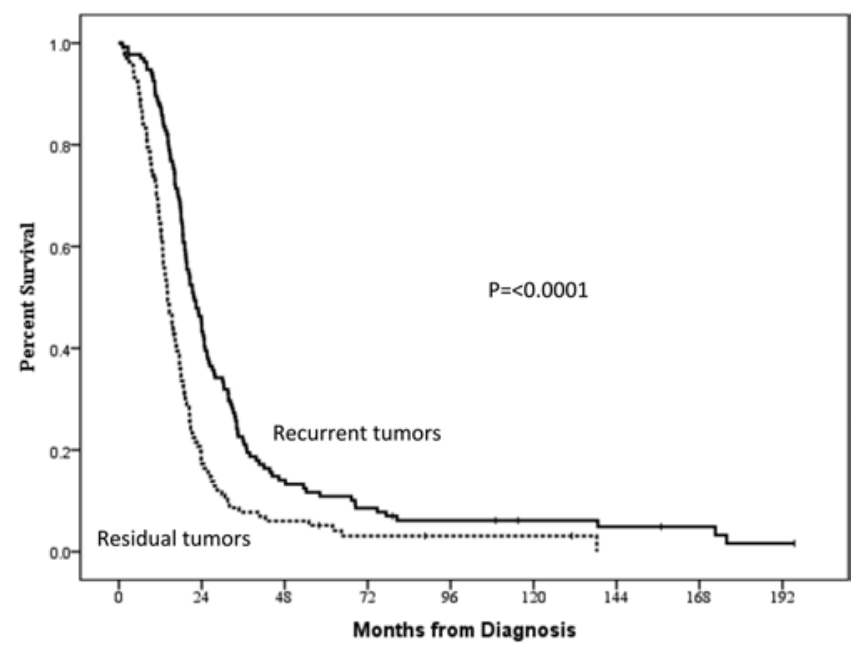

FIG. 1. Kaplan-Meier estimate of OS after diagnosis comparing patients treated with radiosurgery for recurrent versus residual tumors. Radiosurgery at the time of recurrence was associated with significantly improved survival $(p<0.0001)$.
$47.8 \%, 23.6 \%, 11.4 \%$, and $8.1 \%$, respectively. The MST for patients with tumor volumes of $14 \mathrm{~cm}^{3}$ or larger was 6.9 months (95\% CI 5.61-8.19). For these patients, the 6-month, 1-year, 2-year, 3-year, and 5-year survival rates from diagnosis were $58.2 \%, 27.7 \%, 9.4 \%, 5.1 \%$, and $0.9 \%$, respectively. Smaller tumor volumes were associated with improved survival after SRS ( $<<0.0001$ ) (Fig. 2).

The MST after SRS for patients who received a tumor margin dose $\geq 15$ Gy was 12 months (95\% CI 9.8-14.2 months) compared to 8.2 months for patients who received a dose $<15$ Gy (95\% CI 7.4-9.0 months). For patients treated with a margin dose of $\geq 15 \mathrm{~Gy}$, the 6-month, 1-year, 2-year, 3-year, and 5-year survival rates after SRS were $76.7 \%, 49.0 \%, 22.6 \%, 13.1 \%$, and $9.5 \%$, respectively. For patients treated with a margin dose $<15$ Gy, the 6-month, 1-year, 2-year, 3-year, and 5-year survival rates after SRS were $65.9 \%, 32.1 \%, 13.3 \%, 5.4 \%$, and $1.6 \%$, respectively. Patients whose tumors received $\geq 15$ Gy at the margin had better survivals $(p=0.001)$.

Median OS was significantly better $(\mathrm{p}=0.023)$ for patients who underwent SRS for progressive tumors (10.2 months [95\% CI 8.2-12.2 months]) than for those who underwent early SRS for residual unresected tumor volumes (8.4 months [95\% CI 7.3-9.5 months]).

Overall survival was better in patients who were treated with temozolomide after radiosurgery than those patients who were not treated with temozolomide or received other chemotherapy agents $(\mathrm{p}=0.025)$. The MST was 19.3 months (95\% CI 9.4-29.1 months) in patients who received temozolomide after radiosurgery and 8.8 months (95\% CI 7.9-9.76 months) in those who were not treated with temozolomide. Overall survival was better (12.6 vs 8.4 months) in patients who received any chemotherapy after SRS than those who did not receive chemotherapy $(\mathrm{p}=0.004)$.

Overall survival was better for patients who were eligible for additional resection after radiosurgery (15.4 months [95\% CI 12.2-18.67 months]) than it was for patients who

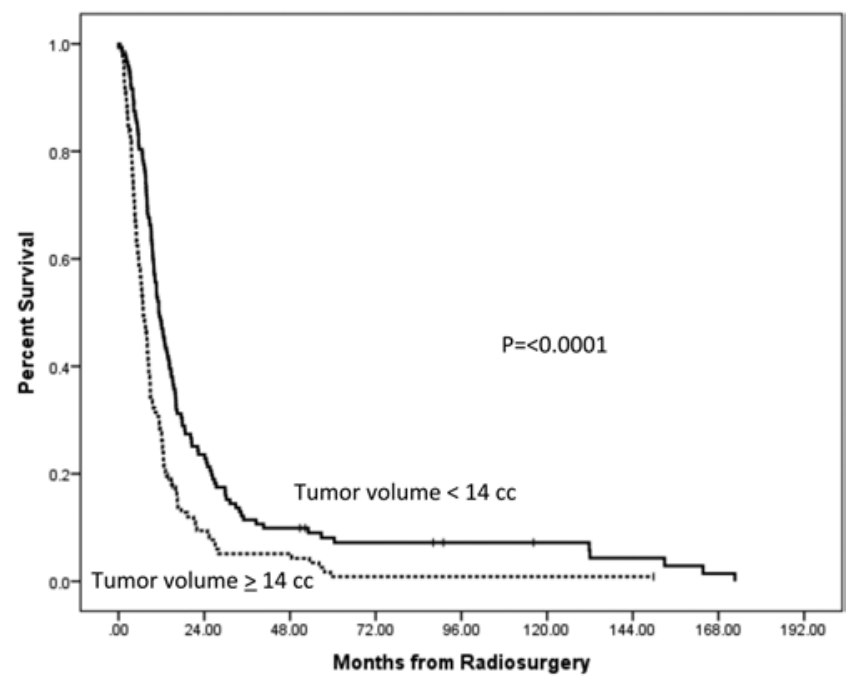

FIG. 2. Kaplan-Meier estimate of OS after radiosurgery comparing patients with tumors smaller than $14 \mathrm{~cm}^{3}$ and patients with tumors $14 \mathrm{~cm}^{3}$ or larger. Smaller tumor volume $\left(<14 \mathrm{~cm}^{3}\right)$ was associated with better OS $(p<0.0001)$. 
were not candidates for additional resection ( 7.5 months [95\% CI 6.6-8.4 months]) ( $\mathrm{p}<0.0001)$.

In the multivariate analysis, factors associated with improved survival after SRS were age $<60$ years $(\mathrm{p}<0.001)$ and tumor volume $<14 \mathrm{~cm}^{3}(\mathrm{p}<0.0005)$ (Table 1$)$.

\section{Progression-Free Survival}

The median PFS was 4.3 months (95\% CI 4.19-5.81). Rates of PFS at 6 months, 1 year, 2 years, 3 years, and 5 years were $45.8 \%, 29.0 \%, 19.2 \%, 15.1 \%$, and $10.6 \%$, respectively. In the univariate analysis a tumor volume $<14$ $\mathrm{cm}^{3}$ was associated with relatively better but clinically insignificant PFS $(p=0.011$ ) (Fig. 3). The median PFS for patients with tumors $<14 \mathrm{~cm}^{3}$ was 4.9 months $(95 \%$ CI 3.08-6.72 months) compared with 4.0 months for patients with tumors $\geq 14 \mathrm{~cm}^{3}$ (95\% CI 3.33-4.67 months).

\section{Imaging Response}

Examination of serial follow-up imaging in 186 patients found that tumor volumes regressed or remained unchanged in 38 patients (20.4\%). However, delayed tumor growth was confirmed in 122 patients (65.6\%), and distant brain spread was detected in 26 patients (14\%). Local tumor control was achieved in $34 \%$ of patients and delayed tumor growth was documented in $65.6 \%$ patients. Examination of histological specimens obtained at the time of repeat resection in 58 patients revealed persistent tumor presence in 49 patients and tumor plus histopathological evidence of radiation effect in a separate 9 patients.

\section{Recursive Partitioning Analysis}

The recursive partitioning analysis (RPA) classification has been advocated for GBM clinical trials to compare trial design and outcomes. Recently $\mathrm{Li}$ et al. revisited this concept and tested a new RPA classification involving 1672 GBM patients from 5 RTOG trials. ${ }^{22}$ These authors proposed a modified model of the RPA classification involving only 4 prognostic factors (age, KPS, extent of resection, and neurological status) for 3 prognostic subgroups of patients with GBM. We used the simplified model of the original RPA classification for our analysis. In the present series the median survival rates for Class III, IV and V+VI were 31.6, 20.8, and 16.7 months, respectively (Table 2). For patients with RPA Class III GBM, 1-year, 2-year, 3-year, and 5-year

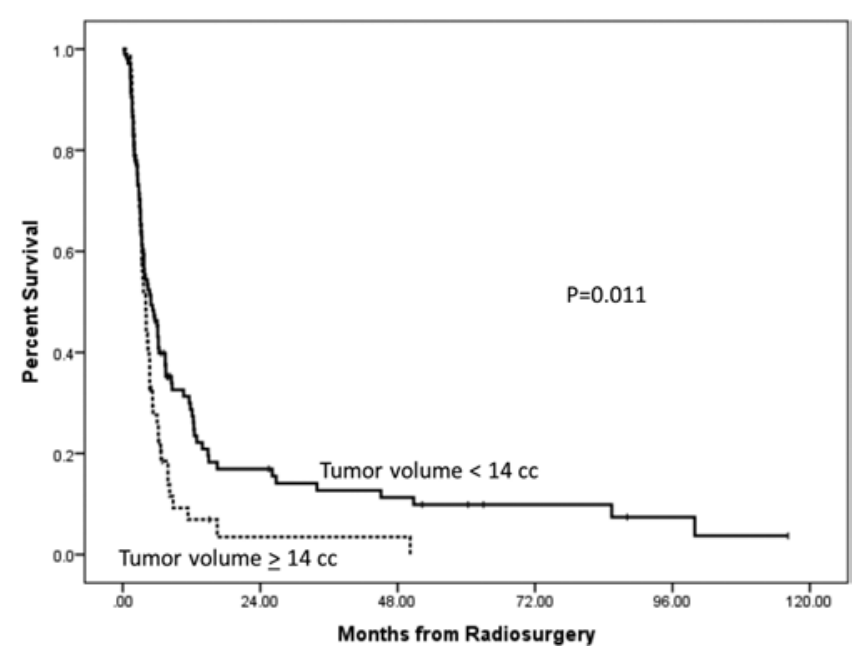

FIG. 3. Kaplan-Meier estimate of PFS after radiosurgery comparing patients with tumors smaller than $14 \mathrm{~cm}^{3}$ and patients with tumors $14 \mathrm{~cm}^{3}$ or larger. Smaller tumor volume $\left(<14 \mathrm{~cm}^{3}\right)$ was associated with better overall PFS $(p=0.011)$.

survival rates were $95.2 \%, 58.2 \%, 39.7 \%$, and $26.4 \%$, respectively. For patients with RPA Class IV GBM, 1-year, 2-year, 3-year, and 5-year survivals were 75\%, 42.5\%, 25\%, and $7.5 \%$, respectively. For patients with RPA Class V+VI GBM, 1-year, 2-year, 3-year, and 5-year survivals were $72.2 \%, 22.1 \%, 7.3 \%$, and $4.3 \%$, respectively. The survival differences among these three classes were statistically significant (Fig. 4).

\section{Adverse Radiation Effects}

Sixty-nine patients (23\%) developed new neurological signs or symptoms associated with imaging changes compatible with AREs. The median time after SRS until the detection of an ARE was 1.7 months. In both univariate and multivariate binary logistic regression models, no variables (dose, tumor volume, timing of SRS compared with RT, and so on) were significantly associated with the development of symptomatic AREs. All patients with suspected AREs received oral corticosteroids. No patient in this series received bevacizumab. Patients with persistent and progressive symptoms underwent resection at the time of progression when their tumor was located in a lobar location.

TABLE 1. Factors associated with overall survival after diagnosis and SRS

\begin{tabular}{|c|c|c|c|c|}
\hline \multirow[b]{3}{*}{ Factor } & \multicolumn{4}{|c|}{$p$ Value } \\
\hline & \multicolumn{2}{|c|}{ OS After Diagnosis } & \multicolumn{2}{|c|}{ Survival After SRS } \\
\hline & Univariate & Multivariate & Univariate & Multivariate \\
\hline Age $<60$ yrs & $<0.0001$ & $<0.0001$ & $<0.00001$ & $<0.0001$ \\
\hline KPS score $\geq 80$ & 0.001 & & 0.048 & 0.296 \\
\hline Gross-total vs subtotal resection & 0.253 & & 0.413 & \\
\hline Prior chemotherapy & $<0.0005$ & 0.001 & 0.175 & \\
\hline Lobar vs deep-seated tumor & 0.007 & & 0.593 & \\
\hline SRS for recurrent vs residual tumor & $<0.0001$ & $<0.0001$ & 0.023 & 0.370 \\
\hline SRS tumor margin dose $\geq 15$ Gy & 0.001 & 0.469 & 0.001 & 0.145 \\
\hline SRS target vol $<14 \mathrm{~cm}^{3}$ & $<0.0001$ & 0.001 & $<0.0001$ & 0.001 \\
\hline
\end{tabular}


TABLE 2. Patient survival after SRS

\begin{tabular}{cccccccc}
\hline \multirow{2}{*}{ Time Frame } & OS After & Survival & Survival for & PFS After & \multicolumn{3}{c}{ Overall Patient Survival } \\
\cline { 7 - 9 } & Diagnosis & After SRS & GBMs $<14 \mathrm{~cm}^{3}$ & SRS & RPA Class III & RPA Class IV & RPA Class V+VI \\
\hline 1 yr & $76.2 \%$ & $37.9 \%$ & $47.8 \%$ & $29.0 \%$ & $95.2 \%$ & $75.0 \%$ & $72.2 \%$ \\
\hline 2 yrs & $30.8 \%$ & $16.7 \%$ & $23.6 \%$ & $19.2 \%$ & $58.2 \%$ & $42.5 \%$ & $22.1 \%$ \\
\hline 3 yrs & $15.1 \%$ & $8.7 \%$ & $11.4 \%$ & $15.1 \%$ & $39.7 \%$ & $25.0 \%$ & $7.3 \%$ \\
\hline 5 yrs & $8.1 \%$ & $4.5 \%$ & $8.1 \%$ & $10.6 \%$ & $26.4 \%$ & $7.5 \%$ & $4.3 \%$ \\
\hline Median (mos) & 18.1 & 9.03 & 11.2 & 4.3 & 31.6 & 20.8 & 16.7 \\
\hline
\end{tabular}

\section{Radiosurgery as Part of Planned Radiosurgical Boost Postirradiation Therapy}

\section{Survival From Diagnosis}

Stereotactic radiosurgery was performed for residual tumors in 144 patients. The MST from the date of diagnosis (on the basis of Kaplan-Meier estimates) was 14.1 months (95\% CI 12.3-15.86 months). Factors associated with better survival included younger age, smaller tumors, use of any chemotherapy, and resection after SRS. The MST for patients younger than 60 years was 16.7 months (95\% CI 12.8-20.5 months). For patients younger than 60 years the 6-month, 1-year, 2-year, 3-year, and 5-year survival rates after SRS were 87.3\%, 72.5\%, 28.5\%, 16.1\%, and $10.7 \%$, respectively. The MST for patients 60 years or older was 13.3 months (95\% CI 11.3.2-15.3 months). For these patients, the 6-month, 1-year, 2-year, 3-year, and 5 -year survival rates from diagnosis were $91.3 \%, 57.5 \%$, $6.8 \%, 0 \%$, and $0 \%$, respectively. Younger age ( $<60$ years) was associated with improved survival after SRS $(\mathrm{p}<$ $0.0001)$ from diagnosis. The MST for patients with tumor volumes < $14 \mathrm{~cm}^{3}$ was 16.7 months (95\% CI 13.5-19.8 months). For patients with tumor volumes $<14 \mathrm{~cm}^{3}$ the 6-month, 1-year, 2-year, 3-year, and 5-year survival rates after SRS were $75.9 \%, 42.1 \%, 21.1 \%, 10.5 \%$, and $2.9 \%$, respectively. The MST for patients with tumor volumes of 14 $\mathrm{cm}^{3}$ or larger was 12.7 months (95\% CI 11.9-13.5 months). For these patients, the 6-month, 1-year, 2-year, 3-year, and

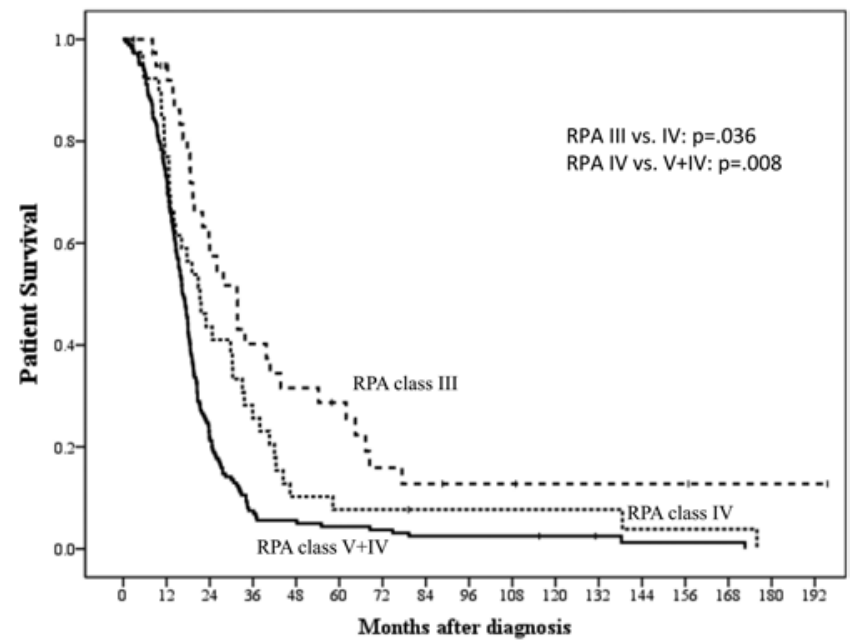

FIG. 4. Kaplan-Meier estimate of OS after diagnosis comparing patients in modified RPA Classes III, IV, and V+VI. Survival was significantly better for patients with RPA Class III GBMs. 5-year survival rates after diagnosis were $58.8 \%, 22.9 \%$, $2.0 \%, 0 \%$, and $0 \%$, respectively. A smaller tumor volume was associated with improved survival after SRS $(\mathrm{p}<$ 0.0001). Overall survival was better for patients who were eligible for additional resection after radiosurgery than for patients who were not candidates for additional resection ( $\mathrm{p}<0.046$ ). The MST for patients who underwent additional resection after radiosurgery was 18.1 months $(95 \%$ CI 14.6-21.6 months) compared with 12.8 months (95\% CI 10.5-15.0 months) for patients who were not eligible to undergo additional resection. Overall survival was better for those who received chemotherapy $(p<0.0001)$. The MST for patients who underwent chemotherapy was 16.6 months (95\% CI 13.6-19.6 months) compared with 11.2 months (95\% CI 8.7-13.6 months) for patients who did not receive chemotherapy.

\section{Survival From SRS}

The overall MST from SRS was 8.4 months (95\% CI 7.3-9.5 months). In the univariate analysis, younger age ( $<60$ years), smaller tumor volume $<14 \mathrm{~cm}^{3}$ ), and resection after SRS were significantly associated with better survival after SRS.

The MST after SRS in patients younger than 60 years was 9.5 months (95\% CI 7.2-11.8). In patients younger than 60 years the 6-month, 1-year, 2-year, 3-year, and 5-year survival rates after SRS were $74.4 \%, 41.9 \%, 26.6 \%$, $14.3 \%$, and $3.4 \%$, respectively. The MST for patients who were 60 years or older was 7.3 months (95\% CI 5.62-8.5). For these patients, the 6-month, 1-year, 2-year, 3-year, and 5 -year survival rates from diagnosis were $60.4 \%, 23.0 \%$, $0 \%, 0 \%$, and $0 \%$, respectively. Younger age ( $<60$ years) was associated with improved survival after SRS $(\mathrm{p}<$ $0.0001)$. The MST for patients with tumor volumes $<14$ $\mathrm{cm}^{3}$ was 10.6 months (95\% CI 8.7-12.4 months). For patients with tumor volumes $<14 \mathrm{~cm}^{3}$ the 6-month, 1-year, 2-year, 3-year, and 5-year survival rates after SRS were $75.9 \%, 42.1 \%, 21.1 \%, 10.5 \%$, and $2.9 \%$, respectively. The MST for patients with tumor volumes of $14 \mathrm{~cm}^{3}$ or larger was 6.9 months (95\% CI 5.68-8.12 months). For these patients, the 6-month, 1-year, 2-year, 3-year, and 5-year survival rates from diagnosis were $58.8 \%, 22.9 \%, 2.0 \%, 0 \%$, and $0 \%$, respectively. Smaller tumor volumes were associated with improved survival after SRS ( $\mathrm{p}<0.0001)$. Overall survival after SRS was better for patients who were eligible for additional resection after radiosurgery than for patients who were not candidates for additional resection $(\mathrm{p}<0.005)$. The MST for patients who underwent addi- 
tional resection after radiosurgery was 12.57 months $(95 \%$ CI 9.8-15.3 months) compared with 7.17 months (95\% CI 6.19-8.14 months) for patients who were not eligible to undergo additional resection.

\section{Progression-Free Survival}

The median PFS was 4.4 months (95\% CI 3.17-5.62). None of the variable tested affected the PFS significantly.

\section{Discussion \\ The Challenge of Glioblastoma}

Glioblastoma multiforme is the most common primary malignant brain tumor in adults. Despite advances in surgical and postoperative radiotherapy techniques, innovative strategies are needed to improve the otherwise dismal prognosis for patients with unresected and progressive tumors. Initial maximal safe cytoreductive surgery in eligible patients (usually those with lobar tumors) improves survival when it is followed by subsequent fractionated radiotherapy and chemotherapy. While data that include all patients with glioblastoma regardless of location have shown little improvement in overall median survival during the last 25 years, carefully selected subgroups may have meaningful improvement in survival. Patients eligible for resection, RT, chemotherapy, repeat resection, and additional multimodality management have increased the rates of longer-term survival.,2,33

After histological diagnosis by biopsy (for deep-seated tumors) or cytoreductive surgery (usually for lobar tumors), fractionated external-beam radiotherapy has become a critical standard of care needed to extend survival. If RT is not added, little benefit from aggressive cytoreductive surgery is realized. In prospective trials its benefit has been a more than doubling of longer-term survival. ${ }^{46}$ During the last 25 years, only two long-term advances have been validated in comprehensive prospective clinical trials. The addition of temozolomide during radiotherapy and for some months thereafter prolonged survival and significantly increased MSTs but only by an average of 2.5 months. ${ }^{44}$ Westphal et al. demonstrated a statistical survival benefit in patients eligible for multimodality management that included reoperation and placement of Gliadel wafers. ${ }^{48}$ The median increase in survival was 1.5 months.

\section{The Potential Benefit of SRS}

Stereotactic radiosurgery is a precise surgical technology that delivers, in a single procedure, a concentrated radiation effect to an imaging-defined target volume. Definition of the target volume-whether the contrast-enhanced volume or the high T2 signal-remains one of the major remaining technical difficulties in the case of malignant glioma. Others have used PET to assist targeting. ${ }^{21} \mathrm{Tu}-$ mor infiltration within the volume of tissue adjacent to the contrast-enhancing volume (estimated by the T2 volume) makes the final target estimate difficult. Using various radiosurgical technologies, prior publications have reported MSTs after glioblastoma radiosurgery that range from 7.5 to 30 months. ${ }^{5,11,12,14,16,26}$ Such variance has led to significant confusion both to the role of SRS and its benefit. The only Level I prospective randomized trial that evaluated
SRS for malignant glioma used a paradigm not commonly employed in practice (i.e., upfront SRS before completion of what is generally thought to be the standard of care: surgery when feasible [otherwise biopsy], fractionated RT, and concomitant carmustine [BCNU] chemotherapy). The radiosurgical methodology in this RTOG trial used both the Gamma Knife and linear accelerators to deliver various SRS doses. This study was not designed to evaluate the role of SRS either early after standard-of-care management or at the time of tumor progression. The treatment paradigm employed in the present report was designed to provide initial phase I/II data related to the use of SRS in the context of current management concepts. The fact that more than 40,000 patients worldwide have undergone glioma SRS for tumor recurrence would support the need for additional knowledge related to its value.

\section{Selection Bias}

Selection bias (both positive and negative) has been implicated as one of the factors that explains the variance in OS after radiosurgery. ${ }^{7,13}$ At our center we strive to identify those patients for whom aggressive multimodality management is likely to have the greatest benefit. In patients eligible for aggressive cytoreductive surgery, fractionated radiotherapy, concomitant temozolomide, and, when possible, additional cytoreductive surgery, the rate of 18-month, 2-year, and 5-year survival has significantly increased. To date, there has been no Level I study that evaluates the benefit of radiosurgery in a clinically relevant paradigm. We believe that the assessment of the role of SRS should be done after standard-of-care radiotherapy and chemotherapy have been delivered. In the present study this included patients who underwent SRS early in the treatment course for unresected tumors or later at the time of tumor progression. We suspect that selection bias improves outcomes in patients eligible for SRS because they have smaller tumor volumes. We believe that it is exactly such patients who should consider the most aggressive interventions and who may gain the greatest dividend. A similar bias exists to define those patients appropriate for surgical resection.

\section{Other SRS Studies for Patients With Glioblastoma}

To study the effect of selection bias, Lustig et al. followed a cohort of patients who were considered eligible for radiosurgery and compared them to a cohort of patients who were not deemed eligible for radiosurgery. These investigators noted no difference in OS between the cohorts. ${ }^{25}$ On the other hand, Mahajan et al. performed a case-control study and concluded that patients with recurrent glioblastoma who undergo SRS require fewer surgical procedures and have longer survivals than do controls. ${ }^{26}$ In contrast, other studies have shown the potential benefit of SRS in the management of recurrent glioblastoma. Kondziolka et al. reported the initial outcome of 64 patients with malignant glioma who underwent radiosurgery at the University of Pittsburgh. ${ }^{14}$ In this study, the MST after radiosurgery was 21 months, and the 2-year survival rate was $51 \%$. An MST of 30 months was noted in patients who underwent SRS at the time of tumor progression. ${ }^{14}$

Pouratian et al. evaluated 48 glioblastoma patients who 
underwent SRS. ${ }^{31}$ Overall MST was 16.2 months. The authors reported that patients treated at the time of progression had significantly longer MSTs than those who were treated as part of the initial treatment paradigm (7.4 vs 15.1 months). ${ }^{31}$ Survival after SRS was not different between the two patient groups. Shrieve et al. reported on 86 patients who underwent linear accelerator-based SRS at the time of tumor recurrence or progression..$^{40}$ The median actuarial survival was 10.2 months and the 1-year and 2-year OS rates were $45 \%$ and $19 \%$, respectively. In this study, age and tumor volume were considered prognostic factors. Villavicencio et al. studied the response to radiosurgery in 36 patients using the CyberKnife in a multicenter study. ${ }^{45}$ The MST after early CyberKnife radiosurgery was 11.5 months compared with 24 months for patients treated at the time of tumor recurrence or progression..$^{45}$

Relatively few studies have attempted to evaluate the value of SRS in combination with adjuvant chemotherapy for recurrent glioblastoma. Larson et al., in a prospective study of SRS in conjunction with the agent Marimastat, showed no survival advantage for patients with recurrent glioblastoma. ${ }^{17}$ Trials have shown that multimodality treatment involving radiosurgery, RT, and chemotherapy is feasible and relatively well tolerated..$^{18,19,35}$

\section{The RPA Classification System}

Curran et al. performed the original RPA of prognostic factors in three RTOG trials more than 2 decades ago. ${ }^{6}$ This analysis included 1578 patients with GBM or anaplastic astrocytoma and generated 6 prognostic classes (Classes I and II for anaplastic astrocytoma, and Classes III-VI for GBM). The reproducibility of this RPA classification system has been verified using cases from the later RTOG trial. ${ }^{36} \mathrm{Li}$ et al. combined Classes V and VI of the original RPA classification because the survivals in these classes were not statistically different. This modified model was easier to apply because it involves only 4 variables: age, KPS, extent of resection, and neurological status. Applying this simplified model to the GBM database that included 1672 GBM patients, these authors reported MSTs of 17.1, 11.2, and 7.5 months for Classes III, IV, and $\mathrm{V}+\mathrm{VI}$, respectively. ${ }^{22}$ In comparison with these historical data, MSTs in the present study were 31.6, 21.3, and 16.7 for Classes III, IV, and V+VI, respectively.

In a recent report, Skeie et al. studied clinical outcomes of 77 patients with recurrent GBM. ${ }^{41}$ Thirty-two patients underwent Gamma Knife SRS, 26 underwent reoperation, and 19 underwent both procedures. These authors showed that patients who underwent SRS had significantly longer survivals than did those who underwent repeat resection. The present study describes our experience in treating a larger number of glioblastoma patients who underwent adjuvant early SRS for residual tumors or later at the time of progression. We noted that the MST after SRS was 9.03 months after radiosurgery and 18.1 months after diagnosis. The 1-year and 2-year OS rates were $37.9 \%$ and $16.7 \%$. The 1-year and 2-year OS rates after diagnosis were $76.2 \%$ and $30.8 \%$. Of patients eligible to undergo this multimodality management strategy, we found that $30 \%$ of those selected for SRS had an OS of 2 years. Adverse radiation effects were noted in $23 \%$ of patients and were generally manageable by a temporary increase in corticosteroid therapy. Our center has also reported the potential benefit of combined SRS and bevacizumab, an agent that may enhance the benefit of SRS and limit the risk of AREs. ${ }^{30}$

\section{Weaknesses of the Present Study}

This study includes a heterogeneous patient population, but it represents a cross-section of the nature of glioblastomas encountered in routine practice. Thus, both patients with lobar tumors eligible for cytoreductive surgery and patients with deep-seated unresectable tumors are included. Many patients received different oncological management therapies during the course of their treatment. Many received chemotherapies as part of clinical trials using drugs that were later shown to be ineffective. It is difficult to sort out the contribution of an individual therapy in patients who undergo multimodal therapies. In addition potential biases that could affect the outcome include selection bias, dose selection based on tumor size, and a tendency to pursue an aggressive management option more often in younger patients with better performance scores.

\section{Conclusions}

This 21-year experience suggests that SRS in carefully selected glioblastoma patients had a favorable survival benefit and was well tolerated. It was associated with a relatively low risk of AREs and represents a noninvasive additional option in a clinical situation in which few options exist. The current study is the largest single-center patient series that examines the role of SRS for glioblastoma, both early after diagnosis for residual unresected tumor and later at the time of tumor progression noted after failure of standard of care management. Important prognostic variables included a tumor volume of less than $14 \mathrm{~cm}^{3}$, a dose at the edge of the tumor volume of $15 \mathrm{~Gy}$ or greater, younger age, and resectable tumor or lobar tumor location.

During this clinical experience, our neurooncology group evaluated numerous chemotherapy and several immunotherapy approaches for GBM as part of both singleand multicenter trials. Most proved ineffective. Given the numerous publications that suggest the benefit of radiosurgery in properly selected patients, we think that brain tumor teams should focus more attention on rigorously evaluating radiosurgery. We believe that a well-designed prospective clinical trial of radiosurgery at the time of tumor recurrence is warranted. A Phase I/II study of SRS and bevacizumab for management of recurrent glioblastoma will begin in 2014 under the auspices of the North American Gamma Knife Consortium.

\section{References}

1. Bleehen NM, Stenning SP: A Medical Research Council trial of two radiotherapy doses in the treatment of grades 3 and 4 astrocytoma. Br J Cancer 64:769-774, 1991

2. Bloch O, Han SJ, Cha S, Sun MZ, Aghi MK, McDermott MW, et al: Impact of extent of resection for recurrent glioblastoma on overall survival. Clinical article. J Neurosurg 117:1032-1038, 2012

3. Butowski N, Lamborn KR, Berger MS, Prados MD, Chang SM: Historical controls for phase II surgically based trials 
requiring gross total resection of glioblastoma multiforme. $\mathbf{J}$ Neurooncol 85:87-94, 2007

4. Cho KH, Hall WA, Lo SS, Dusenbery KE: Stereotactic radiosurgery versus fractionated stereotactic radiotherapy boost for patients with glioblastoma multiforme. Technol Cancer Res Treat 3:41-49, 2004

5. Combs SE, Widmer V, Thilmann C, Hof H, Debus J, SchulzErtner D: Stereotactic radiosurgery (SRS): treatment option for recurrent glioblastoma multiforme (GBM). Cancer 104:2168-2173, 2005

6. Curran WJ Jr, Scott CB, Horton J, Nelson JS, Weinstein AS, Fischbach AJ, et al: Recursive partitioning analysis of prognostic factors in three Radiation Therapy Oncology Group malignant glioma trials. J Natl Cancer Inst 85:704-710, 1993

7. Curran WJ Jr, Scott CB, Weinstein AS, Martin LA, Nelson JS, Phillips TL, et al: Survival comparison of radiosurgeryeligible and -ineligible malignant glioma patients treated with hyperfractionated radiation therapy and carmustine: a report of Radiation Therapy Oncology Group 83-02. J Clin Oncol 11:857-862, 1993

8. Filippini G, Falcone C, Boiardi A, Broggi G, Bruzzone MG, Caldiroli D, et al: Prognostic factors for survival in 676 consecutive patients with newly diagnosed primary glioblastoma. Neuro Oncol 10:79-87, 2008

9. Gannett D, Stea B, Lulu B, Adair T, Verdi C, Hamilton A: Stereotactic radiosurgery as an adjunct to surgery and external beam radiotherapy in the treatment of patients with malignant gliomas. Int J Radiat Oncol Biol Phys 33:461-468, 1995

10. Gorlia T, van den Bent MJ, Hegi ME, Mirimanoff RO, Weller $\mathrm{M}$, Cairncross JG, et al: Nomograms for predicting survival of patients with newly diagnosed glioblastoma: prognostic factor analysis of EORTC and NCIC trial 26981-22981/CE.3. Lancet Oncol 9:29-38, 2008

11. Hall WA, Djalilian HR, Sperduto PW, Cho KH, Gerbi BJ, Gibbons JP, et al: Stereotactic radiosurgery for recurrent malignant gliomas. J Clin Oncol 13:1642-1648, 1995

12. Hsieh PC, Chandler JP, Bhangoo S, Panagiotopoulos K, Kalapurakal JA, Marymont MH, et al: Adjuvant gamma knife stereotactic radiosurgery at the time of tumor progression potentially improves survival for patients with glioblastoma multiforme. Neurosurgery 57:684-692, 2005

13. Irish WD, Macdonald DR, Cairncross JG: Measuring bias in uncontrolled brain tumor trials - to randomize or not to randomize? Can J Neurol Sci 24:307-312, 1997

14. Kondziolka D, Flickinger JC, Bissonette DJ, Bozik M, Lunsford LD: Survival benefit of stereotactic radiosurgery for patients with malignant glial neoplasms. Neurosurgery 41:776-785, 1997

15. Kondziolka D, Lunsford LD, Flickinger JC: In regard to Dr. Souhami et al. (Int J Radiat Oncol Biol Phys 2004;60:853860). Int J Radiat Oncol Biol Phys 62:614-616, 2005 (Letter)

16. Larson DA, Gutin PH, McDermott M, Lamborn K, Sneed PK, Wara WM, et al: Gamma knife for glioma: selection factors and survival. Int J Radiat Oncol Biol Phys 36:10451053,1996

17. Larson DA, Prados M, Lamborn KR, Smith V, Sneed PK, Chang S, et al: Phase II study of high central dose Gamma Knife radiosurgery and marimastat in patients with recurrent malignant glioma. Int J Radiat Oncol Biol Phys 54:13971404,2002

18. Lederman G, Arbit E, Odaimi M, Wertheim S, Lombardi E: Recurrent glioblastoma multiforme: potential benefits using fractionated stereotactic radiotherapy and concurrent taxol. Stereotact Funct Neurosurg 69:162-174, 1997

19. Lederman G, Wronski M, Arbit E, Odaimi M, Wertheim S, Lombardi E, et al: Treatment of recurrent glioblastoma multiforme using fractionated stereotactic radiosurgery and concurrent paclitaxel. Am J Clin Oncol 23:155-159, 2000

20. Lee SW, Fraass BA, Marsh LH, Herbort K, Gebarski SS, Martel MK, et al: Patterns of failure following high-dose 3-D conformal radiotherapy for high-grade astrocytomas: a quantitative dosimetric study. Int J Radiat Oncol Biol Phys 43:79-88, 1999

21. Levivier M, Massager N, Wikler D, Devriendt D, Goldman $\mathrm{S}$ : Integration of functional imaging in radiosurgery: the example of PET scan. Prog Neurol Surg 20:68-81, 2007

22. Li J, Wang M, Won M, Shaw EG, Coughlin C, Curran WJ Jr, et al: Validation and simplification of the Radiation Therapy Oncology Group recursive partitioning analysis classification for glioblastoma. Int J Radiat Oncol Biol Phys 81:623-630, 2011

23. Loeffler JS, Alexander E III, Shea WM, Wen PY, Fine HA, Kooy HM, et al: Radiosurgery as part of the initial management of patients with malignant gliomas. J Clin Oncol 10:1379-1385, 1992

24. Loeffler JS, Alexander E III, Wen PY, Shea WM, Coleman $\mathrm{CN}$, Kooy HM, et al: Results of stereotactic brachytherapy used in the initial management of patients with glioblastoma. J Natl Cancer Inst 82:1918-1921, 1990

25. Lustig RA, Scott CB, Curran WJ: Does stereotactic eligibility for the treatment of glioblastoma cause selection bias in randomized studies? Am J Clin Oncol 27:516-521, 2004

26. Mahajan A, McCutcheon IE, Suki D, Chang EL, Hassenbusch SJ, Weinberg JS, et al: Case-control study of stereotactic radiosurgery for recurrent glioblastoma multiforme. J Neurosurg 103:210-217, 2005

27. Mehta MP, Masciopinto J, Rozental J, Levin A, Chappell R, Bastin K, et al: Stereotactic radiosurgery for glioblastoma multiforme: report of a prospective study evaluating prognostic factors and analyzing long-term survival advantage. Int J Radiat Oncol Biol Phys 30:541-549, 1994

28. Nagai H, Kondziolka D, Niranjan A, Flickinger JC, Lunsford LD: Results following stereotactic radiosurgery for patients with glioblastoma multiforme. Radiosurgery 5:91-99, 2004

29. Nwokedi EC, DiBiase SJ, Jabbour S, Herman J, Amin P, Chin LS: Gamma knife stereotactic radiosurgery for patients with glioblastoma multiforme. Neurosurgery 50:41-47, 2002

30. Park KJ, Kano H, Iyer A, Liu X, Niranjan A, Flickinger JC, et al: Salvage gamma knife stereotactic radiosurgery followed by bevacizumab for recurrent glioblastoma multiforme: a case-control study. J Neurooncol 107:323-333, 2012

31. Pouratian N, Crowley RW, Sherman JH, Jagannathan J, Sheehan JP: Gamma Knife radiosurgery after radiation therapy as an adjunctive treatment for glioblastoma. J Neurooncol 94:409-418, 2009

32. Prisco FE, Weltman E, de Hanriot RM, Brandt RA: Radiosurgical boost for primary high-grade gliomas. J Neurooncol 57:151-160, 2002

33. Sanai N, Polley MY, McDermott MW, Parsa AT, Berger MS: An extent of resection threshold for newly diagnosed glioblastomas. J Neurosurg 115:3-8, 2011

34. Sarkaria JN, Mehta MP, Loeffler JS, Buatti JM, Chappell RJ, Levin AB, et al: Radiosurgery in the initial management of malignant gliomas: survival comparison with the RTOG recursive partitioning analysis. Int J Radiat Oncol Biol Phys 32:931-941, 1995

35. Schwer AL, Damek DM, Kavanagh BD, Gaspar LE, Lillehei K, Stuhr K, et al: A phase I dose-escalation study of fractionated stereotactic radiosurgery in combination with gefitinib in patients with recurrent malignant gliomas. Int $\mathbf{J}$ Radiat Oncol Biol Phys 70:993-1001, 2008

36. Scott CB, Scarantino C, Urtasun R, Movsas B, Jones CU, Simpson JR, et al: Validation and predictive power of Radiation Therapy Oncology Group (RTOG) recursive partitioning 
analysis classes for malignant glioma patients: a report using RTOG 90-06. Int J Radiat Oncol Biol Phys 40:51-55, 1998

37. Selker RG, Shapiro WR, Burger P, Blackwood MS, Arena VC, Gilder JC, et al: The Brain Tumor Cooperative Group NIH Trial 87-01: a randomized comparison of surgery, external radiotherapy, and carmustine versus surgery, interstitial radiotherapy boost, external radiation therapy, and carmustine. Neurosurgery 51:343-357, 2002

38. Shenouda G, Souhami L, Podgorsak EB, Bahary JP, Villemure JG, Caron JL, et al: Radiosurgery and accelerated radiotherapy for patients with glioblastoma. Can J Neurol Sci 24:110-115, 1997

39. Shrieve DC, Alexander E III, Black PM, Wen PY, Fine HA, Kooy HM, et al: Treatment of patients with primary glioblastoma multiforme with standard postoperative radiotherapy and radiosurgical boost: prognostic factors and long-term outcome. J Neurosurg 90:72-77, 1999

40. Shrieve DC, Alexander E III, Wen PY, Fine HA, Kooy HM, Black PM, et al: Comparison of stereotactic radiosurgery and brachytherapy in the treatment of recurrent glioblastoma multiforme. Neurosurgery 36:275-284, 1995

41. Skeie BS, Enger PO, Brøgger J, Ganz JC, Thorsen F, Heggdal $\mathrm{JI}$, et al: Gamma knife surgery versus reoperation for recurrent glioblastoma multiforme. World Neurosurg 78:658669, 2012

42. Souhami L, Seiferheld W, Brachman D, Podgorsak EB, Werner-Wasik M, Lustig R, et al: Randomized comparison of stereotactic radiosurgery followed by conventional radiotherapy with carmustine to conventional radiotherapy with carmustine for patients with glioblastoma multiforme: report of Radiation Therapy Oncology Group 93-05 protocol. Int J Radiat Oncol Biol Phys 60:853-860, 2004

43. Stummer W, Pichlmeier U, Meinel T, Wiestler OD, Zanella F, Reulen HJ: Fluorescence-guided surgery with 5-aminolevulinic acid for resection of malignant glioma: a randomised controlled multicentre phase III trial. Lancet Oncol 7:392 401, 2006
44. Stupp R, Mason WP, van den Bent MJ, Weller M, Fisher $\mathrm{B}$, Taphoorn MJ, et al: Radiotherapy plus concomitant and adjuvant temozolomide for glioblastoma. $\mathbf{N}$ Engl J Med 352:987-996, 2005

45. Villavicencio AT, Burneikiene S, Romanelli P, Fariselli L, McNeely L, Lipani JD, et al: Survival following stereotactic radiosurgery for newly diagnosed and recurrent glioblastoma multiforme: a multicenter experience. Neurosurg Rev 32:417-424, 2009

46. Walker MD, Strike TA, Sheline GE: An analysis of doseeffect relationship in the radiotherapy of malignant gliomas. Int J Radiat Oncol Biol Phys 5:1725-1731, 1979

47. Wallner KE, Galicich JH, Krol G, Arbit E, Malkin MG: Patterns of failure following treatment for glioblastoma multiforme and anaplastic astrocytoma. Int J Radiat Oncol Biol Phys 16:1405-1409, 1989

48. Westphal M, Hilt DC, Bortey E, Delavault P, Olivares R, Warnke PC, et al: A phase 3 trial of local chemotherapy with biodegradable carmustine (BCNU) wafers (Gliadel wafers) in patients with primary malignant glioma. Neuro Oncol 5:79-88, 2003

\section{Author Contributions}

Conception and design: Niranjan. Acquisition of data: Iyer. Analysis and interpretation of data: Niranjan, Kano, Flickinger. Drafting the article: Niranjan, Lunsford. Critically revising the article: all authors. Reviewed submitted version of manuscript: all authors. Approved the final version of the manuscript on behalf of all authors: Niranjan.

\section{Correspondence}

Ajay Niranjan, Department of Neurological Surgery, University of Pittsburgh, Ste. B-400, UPMC Presbyterian, 200 Lothrop St., Pittsburgh, PA 15213. email: niranjana@upmc.edu. 